* Corresponding author

E-mail address: milan.ruzicka@fs.cvut.cz (M. Růžička)

Article information

Article history: Volume15-No. 2-00106-11

Received 28 March 2011

Accepted 24 May 2011

Available online 26 August

\section{Fatigue Behaviour of Laser Machined Aluminium Alloy 2024-T3}

\author{
Růžička Milan a* , Doubrava Karel ${ }^{\text {a }}$, Vargas Miguel ${ }^{\mathrm{a}}$, Kramberger Janez ${ }^{\mathrm{b}}$ \\ a Czech Technical University in Prague, Faculty of Mechanical Engineering, Technická 4, 16607 Praha6, Czech Republic \\ ${ }^{b}$ Faculty of Mechanical Engineering, University of Maribor, Smetanova 17, SI-2000 Maribor, Slovenia
}

\title{
BIOGRAPHICAL NOTES
}

Milan Růžička, prof. Ing. CSc. (born 1955) works at the Faculty of Mechanical Engineering of the Czech Technical University in Prague (FME CTU). He finished his engineering study in 1979, CSc (PhD) degree in 1984. He is the head of Division of Strength of materials and professor since 2005. During his research collaboration he visited and worked with the Aeronautical Research Institute (VZLÚ) in Prague, the University in Karlsruhe, Germany (1987), University in Stuttgart and TU Darmstadt (1992), Institute of thermo mechanics Czech Academy of Science (1995-1997), Stanford University USA (2004). His research area is focused on applied mechanics, limit states of structures, experimental analysis of stress and strain in structures, mechanical and fatigue properties of metals and composites, fatigue life prediction and reliability. He is member of the scientific committee of the Danubia-Adria Society on Advances in Experimental Mechanics, the Czech Society for Mechanics, the Society for Biomechanics and the Czech Engineering Academy. Prof. Ruzicka is the author or co-author of more than 25 journal publications, 40 publications and contributions to international conferences, 4 univ. text books and 3 monographs.

Karel Doubrava, Ing. Ph.D. (born 1975) works as senior assistant at Faculty of Mechanical Engineering of Czech Technical University in Prague (FME CTU). He finished his master degree in 1998 and his Ph.D. in 2006. His research activities are focused on stress and strength of materials, experimental static stress analysis and fatigue testing of materials and structures.

Miguel Vargas, BSc. MSc. (born 1978) has engineering degree from the University of Santiago de Chile, 2003. He studied metallurgical engineering-study field: Metallurgy of steels and ferroalloys. 2002/03 he stayed at DAAD exchange program on the: Institute for Steelmaking of the Technical University of Aachen, Germany. From 2009 he is the PhD student at FME CTU in Prague.

Janez Kramberger, BSc. MSc. PhD. (born 1963) works as Assistant Professor and Senior Researcher at the Faculty of Mechanical Engineering of the University of Maribor, Slovenia. He finished Master of Science in Mechanical engineering at University of Maribor in 1990 and reached the title Doctor of Technical Sciences at University of Maribor in 2001. During his research and grant activities he visited and worked at the Wessex Institute of technology, Southampton, UK, (1994) and the Technical University of Munich (2002). He is member of Slovenian Society of Mechanics, Society of University Teachers, and Member of Technical committee for standards. His professional research interest is focused on Computational mechanics, Fracture mechanics, Fatigue problems, Design of welded structures. He have published more as 20 papers in national and international journals. 


\section{KEY WORDS}

Laser Cutting, Fatigue Life, Aluminum Alloy 2024-T3

\section{ABSTRACT}

The advantages offered by the laser technology to cut materials are very attractive for the manufacturing industry. This is due to its versatility and to the fact that they can be faster and cleaner than conventional machining techniques, leaving edges with a high quality surface finish. Nevertheless, this technology cuts the material either melting it, vaporizing it away or blowing it away by a jet of gas. It causes changes on and around the cut off zone and therefore changes in the mechanical response for the whole part. Geometric microstructure of the cut surface content micronotches, where fatigue cracks can nucleate. Considering this, the study of the influence of the laser optical power (speed of a cutting) on the fatigue behavior of laser machined 2024-T3 Alloy sheet was examined. Specimens of different thickness from 2 to $6 \mathrm{~mm}$ have been prepared by $\mathrm{CO}_{2}$ laser cutting machine. The results show that the cutting speed must be optimized for different thickness of a plate. Surface flaws have a determinant influence on the fatigue life behavior.

\section{Introduction}

The laser cutting offers several advantages as compared to other slitting processes such as plasma cutting, punching and nibbling or wire erosion [2]:

- Processing the workpiece is possible without contact or force.

- As opposed to punching and nibbling, almost every contour shape can be made, without requiring a single tool change.

- With the laser beam, both large cuts in any shape can be cut as well as small, filigree and complicated contours. Geometric shapes can be processed especially quickly with only a few piercings.

- Separation is precise. The extremely narrow kerf remains virtually constant. Tolerances up to $0.05 \mathrm{~mm}$ can be maintained in the series production.

- The cutting speed is high. Therefore, the course of production can be considerably accelerated as compared to wire erosion.

Due to the high energy density, the heat-affected zone can be kept very small: hardening depths from 0.1 to $0.2 \mathrm{~mm}$ are possible. An oxide film forms when cutting with oxygen.

- Less heating of materials ensures less distortion of the material at all events.
The roughness of the cutting surfaces is low: less than $100 \mu \mathrm{m}$. The workpiece does not require refinishing.

- The most commonly used steels can be cut without any burr formation, eliminating the need for subsequent burr removal.

\section{Laser Cutting}

What happens when a laser beam is used on material? If a laser beam is used on a workpiece, the material heats up so much that it melts or evaporates. The cutting process begins if it has penetrated the workpiece completely. The laser beam moves along the part contour and melts the material continuously, see Fig. 1. It is blown from the kerf with the help of a gas current. A narrow kerf between the part and the waste grid occurs. The cutting gas current exits the nozzle with the laser beam.
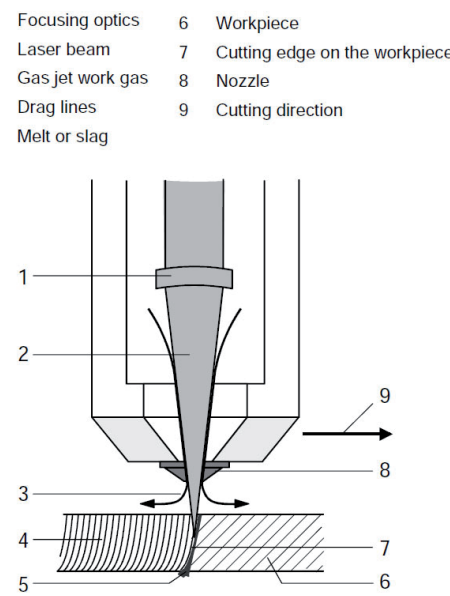

Fig. 1: Principle of a laser cutting

\section{Cutting quality criteria}

Cutting criteria such as pitting, burr or groove coasting, see Fig. 2, can be analyzed with the naked eye. Additional equipment is used for the roughness, the perpendicularly and the width of gap. Which criteria are important and when, must be practically determined. The function of the part is decisive here.

When cutting the contours using the laser beam, perpendicular drag lines are formed on the cutting surfaces. Its depth determines the roughness. The lesser the roughness, more even is the cutting surface. During laser cutting using $\mathrm{CO}_{2}$ lasers, the roughness increases with the material thickness. A kerf oc- 
curs during laser cutting, which is usually not parallel from the upper cutting edge to the lower cutting edge.

The kerf width increases with the material thickness. Its width and shape (Fig. 3) depends on the following factors:

\section{- Focal diameter, \\ - Material, \\ -Wavelength, \\ - Cutting procedure.}

Criteria for the Quality of the Cutting Edge

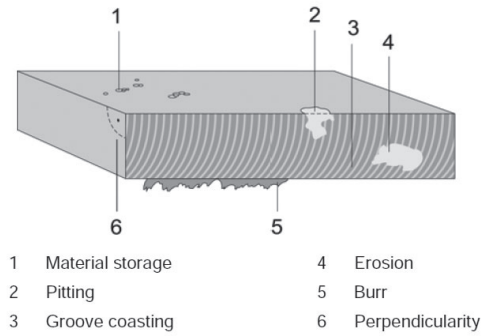

Fig. 2: Main considerations to evaluate a cutting edge.

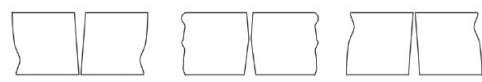

Fig. 3: Kerf shapes generated by laser cutting.

It varies between $0.15 \mathrm{~mm}$ (material thicknesses of $1-6 \mathrm{~mm}$ ) and up to $0.5 \mathrm{~mm}$ (material thicknesses of $20-30 \mathrm{~mm}$ ). The width must be constant over the entire work area; otherwise, the dimensions of the parts and contours become inaccurate.

Burr-freeness is one of the most important criteria for laser cuts. This is so because burr must be always removed. Several different burrs can be formed: right from crumbly slag residue that can be easily removed up to sharp metallic burr that is firmly stuck to the lower side of the edge.

\section{Cutting Power and Cutting Speed}

The laser power must be adapted to the procedure, the type of material and the material thickness. The maximum material thickness, that can be cut, depends on the type of material and the laser power. Generally: The material thickness increases with the laser power, which can be still processed.

Like the laser power, even the cutting speed must be adapted to the type of material and the material thickness. An erroneous cutting speed can lead to roughness, burr formation or pits in the cut contour. If the contour is cut too quickly, then it might result in the material not slitting any longer. Basically: The higher the laser power available, faster is the cutting. In case of equal laser power, the cutting speed decreases with increasing material thickness. Optimization of cutting parameters was the main objective of this research.

\section{Experimental Procedure}

The studied samples were made from Al-alloy 2024 plates. The chemical composition of these samples is given in the Table 1, (ASM, http://asm.matweb. com). Theses plates were thermally treated according to $\mathrm{T} 3$ treatment, which consists in solution immersion, cold wrought hardening and natural aging.

Dog bone specimens of $220 \mathrm{~mm}$ length, $80 \mathrm{~mm}$ width and with the notch radius of $100 \mathrm{~mm}$ were prepared, as shown in the Fig. 4.

Table 1: Chemical composition of aluminum 2024 plates [\%].

\begin{tabular}{|c|c|c|c|c|}
\hline $\mathbf{A L}$ & $\mathbf{C u}$ & $\mathbf{M g}$ & $\mathbf{M n}$ & $\mathbf{F e}$ \\
\hline $90.7-94.7$ & $3.8-4.9$ & $1.2-1.8$ & $0.3-0.9$ & $\max 0.5$ \\
\hline
\end{tabular}

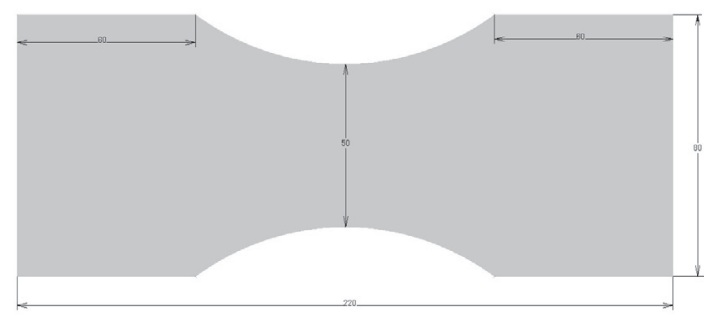

Fig. 4: Sample geometry.

The tests were carried out for 4 different nominal thickness series $(2.0,2.5,3.0$ and $6.0 \mathrm{~mm})$ at 4 cutting speeds. The nominal laser cutting power was 5 [kW]. The fatigue test machine used was resonant pulsator Amsler type 10HFP (maximum of force $100 \mathrm{kN}$ ), with a working frequency about $90 \mathrm{~Hz}$ under standard room temperature (23 degrees of Celsius) and standard pressure conditions.

Considering that the nominal fatigue stress for this alloy is about $130 \mathrm{MPa}, 2$ stress levels in the high cycle range were chosen to test the samples: 115 and $170 \mathrm{MPa}$. Here we will comment results on the stress level $170 \mathrm{MPa}$. Six samples were tested for thickness series (2.0, 2.5, 3.0 and $6.0 \mathrm{~mm})$.

The fatigue test was conducted using a stress ratio 
$R=0.05$, with constant stress amplitude. Tests were stopped automatically when the frequency fall down about $10 \%$, it represent cracks between $5-10 \mathrm{~mm}$.

\section{Statistical Analysis}

The data analysis was carried out using a statistical evaluation method, (ISO 3800:1993). A log-normal statistical distribution is suggested and therefore the number of cycles was converted to 10 base logarithmic scale and sort from lower to higher values.

The mean values and scatters of experimental results in the finite life range can be observed in a diagram with the nonlinear probability scale. By logarithmic transformation, the experimental points in probability graph determine straight line in basis the normal distribution of $\log N$ and using the sorted ordinal probability estimator (1):

$$
P_{i}=(3 i-1) /(3 n+1)
$$

where: $P_{i}$ - is the probability of failure in the finite life range; $i$ - is the ordinal number of a test piece and $n$ - is the number of test pieces tested.

The procedure can be explained with an example: Six samples of the nominal thickness $2.0 \mathrm{~mm}$ were cut with the cutting speed $20[\mathrm{~m} / \mathrm{s}]$ and were tested on constant stress amplitude $\sigma_{\mathrm{a}}=170 \mathrm{MPa}$. The samples reached the failure at: $N=(93441,70352$, $68870,68955,83253,92815)$ cycles. Now the number of cycles is arranged according to size in logarithmic scale and ordinals probability $P_{i}$ are assigned to them. All values are sorted in the Table 2. Finally, the estimation of the mean value (2), variation (3) and standard deviation (4) according next relations were calculated:

$$
\begin{aligned}
\hat{\mu} & =\bar{x}=\frac{\sum_{i} \log N_{i}}{n} \\
\hat{s}^{2} & =\frac{\sum_{i}\left(\bar{x}-\log N_{i}\right)^{2}}{n-1}
\end{aligned}
$$

and

$$
\hat{s}=K \cdot \sqrt{\hat{s}^{2}}
$$

where $K$ is a correction parameter that depends on the sample size $\mathrm{n}$ as follows [3]:

$$
\begin{aligned}
K & =\sqrt{\frac{2}{n-1}} \frac{\Gamma\left(\frac{n}{2}\right)}{\Gamma\left(\frac{n-1}{2}\right)} \\
& =1-\frac{1}{4 n}-\frac{1}{32 n^{2}}-\ldots
\end{aligned}
$$

Here $\Gamma$ is the gamma function. As $n$ grows up, $K$ approaches 1 , but for smaller sample size $\mathrm{n}$ the correction must be taken in the account.

The Table 3 below gives the parameter $K$ for a few values of $n$ :

Table 3: Correction parameter $K$.

\begin{tabular}{|c|c|c|}
\hline Sample size $\mathbf{n}$ & $\begin{array}{c}\text { Expression of } \\
\text { factor K eq. (5) }\end{array}$ & $\begin{array}{c}\text { Num. } \\
\text { aproximation }\end{array}$ \\
\hline 4 & $2 \cdot \sqrt{\frac{2}{3 \pi}}$ & 0.9213 \\
\hline 5 & $\frac{3}{4} \cdot \sqrt{\frac{\pi}{2}}$ & 0.9400 \\
\hline 6 & $\frac{8}{3} \cdot \sqrt{\frac{2}{5 \pi}}$ & 0.9515 \\
\hline
\end{tabular}

Also a slope of the scatter can be drawn for each series in a nonlinear probabilistic scale. Nevertheless this hasn't been drawn using linear regresion tools, but with the help of the standard deviation. After that we calculate $(-1 \mathrm{~s})$ and $(+1 \mathrm{~s})$ for each series values and we plotted these points against their respective estimated fatigue life data.

Looking the draws, which are depicted on the Fig. 5 to Fig. 8, we can see that most of the mean values (for probability of fracture of $50 \%$ ) lie on the range of 4.9 to 5.5 of the abscissa axis, which expressed back to cycles gives 79400 as the lowest value versus 316200 for the highest one. It means that influence of cutting speed and thickness on the final fatigue life is significant for practical application.

Table 2: Example of the results procedure.

\begin{tabular}{c|c|c|c|c|c|c|c|}
\hline Ordinal & $\mathbf{1}$ & $\mathbf{3}$ & $\mathbf{3}$ & $\mathbf{4}$ & \multicolumn{1}{c|}{$\mathbf{6}$} \\
\hline Cycles $\left[\log \mathbf{N}_{\mathbf{i}}\right]$ & 4.83 & 4.84 & 4.85 & 4.19 & 4.96 & 4.97 \\
\hline $\mathbf{P}_{\mathbf{i}}[\%]$ & 10.53 & 26.32 & 42.11 & 57.89 & 73.68 & 89.4 \\
\hline
\end{tabular}


After all data was plotted, the highest fatigue life of each thickness series was chosen and drawn back together on single charts. As a tendency was found, that the best values correspond to the lowest cutting speeds in almost all thickness series, the only one exception is for the $2.0 \mathrm{~mm}$ thickness series processed

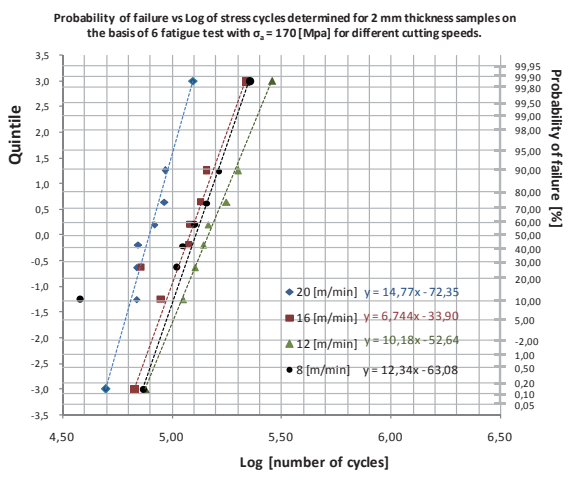

Fig. 5: Probability of failure versus log cycles for $2.0 \mathrm{~mm}$ nominal tickness.

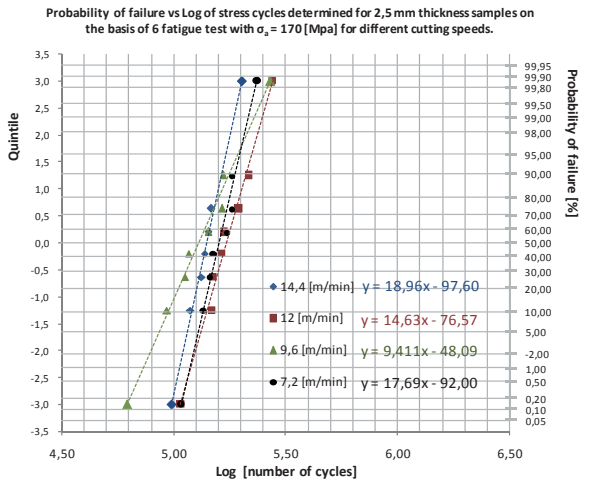

Fig. 6: Probability of failure versus log cycles for $2.5 \mathrm{~mm}$ nominal thickness.

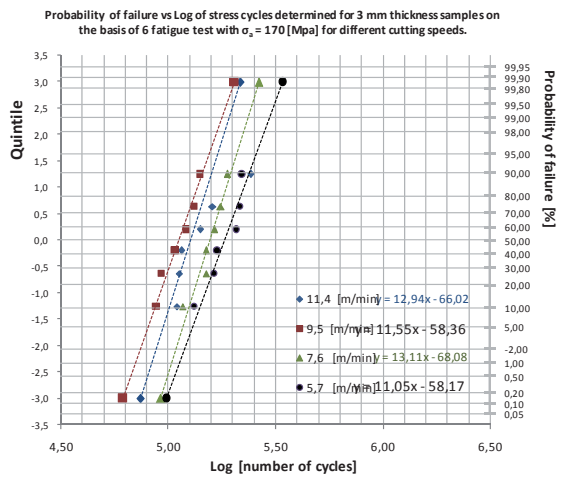

Fig. 7: Probability of failure versus log cycles for $3.0 \mathrm{~mm}$ nominal thickness. at $12[\mathrm{~m} / \mathrm{min}]$ of the cutting speed.

Such comparison can be seen on the Fig. 9. Here is clear to see that the $6.0 \mathrm{~mm}$ thick specimens have an significant higher fatigue life as give results for relative thinner plates between 2.0 to $3.0 \mathrm{~mm}$.

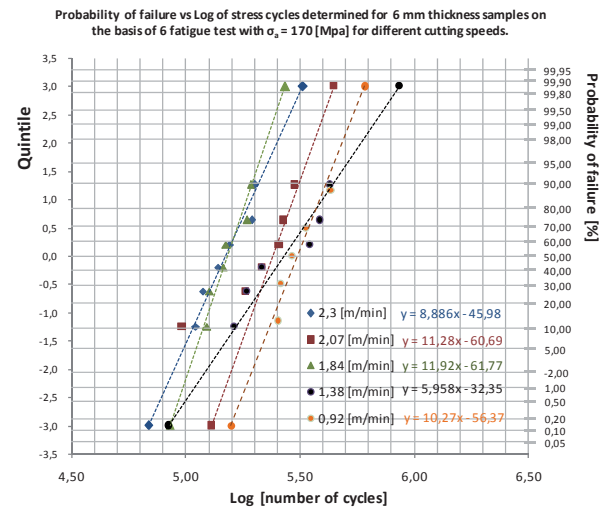

Fig. 8: Probability of failure versus $l o g$ cycles for $6.0 \mathrm{~mm}$ nominal thickness.

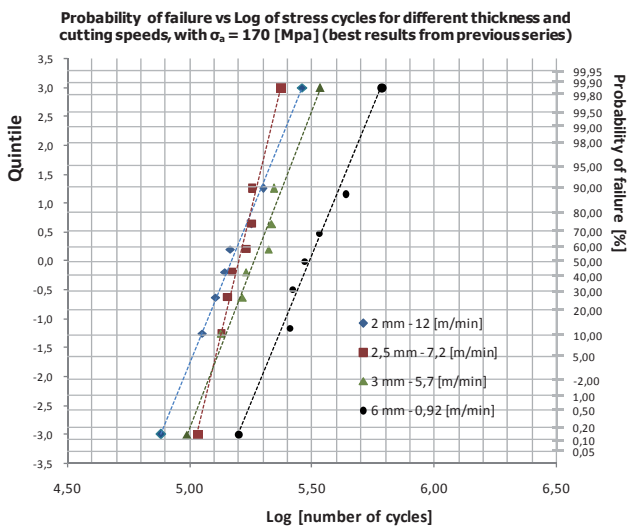

Fig. 9: Best Probability failure results versus log cycles from all thickness series at 170 [MPa].

\section{Surface inspection of the laser processed samples}

With the help of an optic microscope we can carry out a surface inspection of the laser processed samples. Fig. 10 shows a micro-photography of a $2.0 \mathrm{~mm}$ thick sample. The image shows a pitting flaw, on which nucleates the fracture crack.

Fig. 11 displays micro-photography of a $6.0 \mathrm{~mm}$ thick sample showing its edge. On it can be seen the striations leaved by the laser cutting beam, which act as stress concentrator. Fig. 12 shows the micro-photography of a $6.0 \mathrm{~mm}$ thick sample showing its edge. Here again can be seen, on a different sample, that the striations act as micro-notch or stress concentrator and collaborate the cracks to nucleate. 


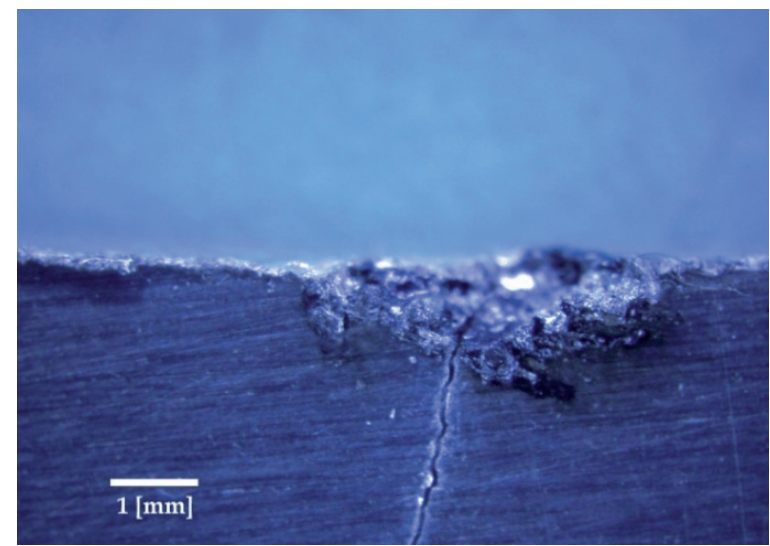

Fig. 10: Micro-photography of a $2.0 \mathrm{~mm}$ thick sample.

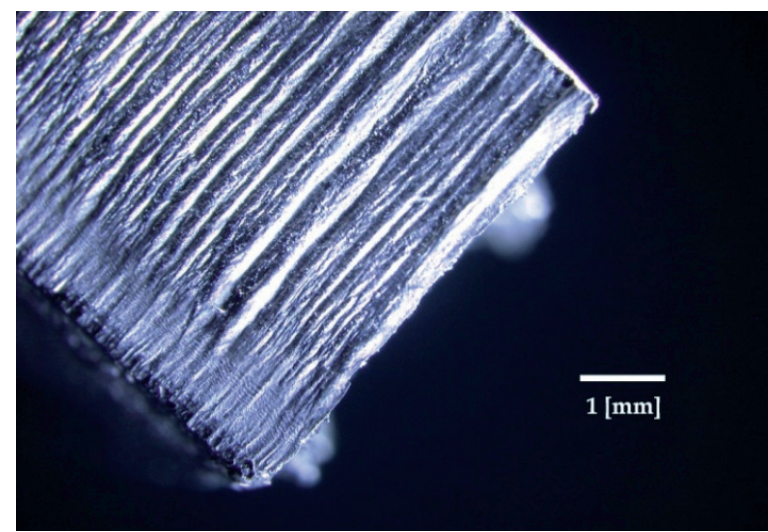

Fig. 11: Micro-photography of a $6.0 \mathrm{~mm}$ thick sample showing its edge.

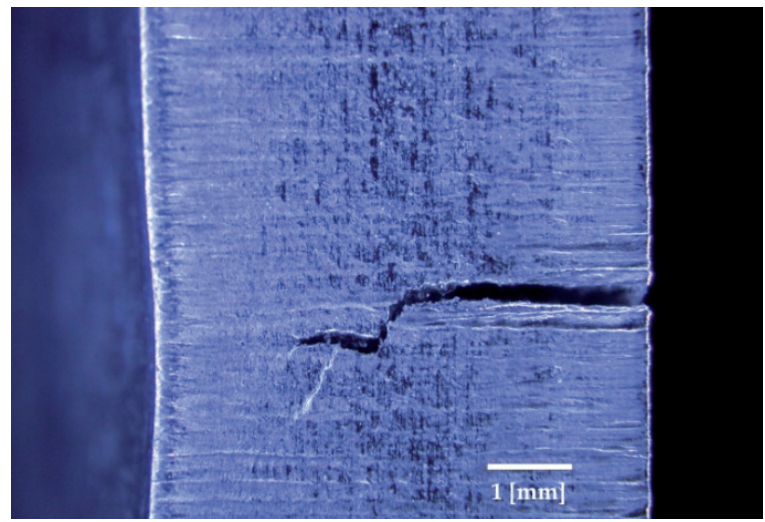

Fig. 12: Micro-photography of a $6.0 \mathrm{~mm}$ thick sample with a crack.

Fig. 13 demonstrates the effect of the over speed processing on a speciment of $2.5 \mathrm{~mm}$ thick. On it we can see three zones: from right to left it can be seen the face on which impacts the laser beam and it cuts around $1 \mathrm{~mm}$ perpendicularly to the sample, then a transition zone, and finally an oblique set of striations due to the excess of speed.

All here presented damages and surface flaws are ineligible for real applications because of reduction of its fatigue life.

After these observations we can infer that some surface errors have a major significance for the fatigue behavior and its presence is particularly harmful in low thickness samples, as we could see in the Fig. 10, where a simple pitting originates the fatigue crack.

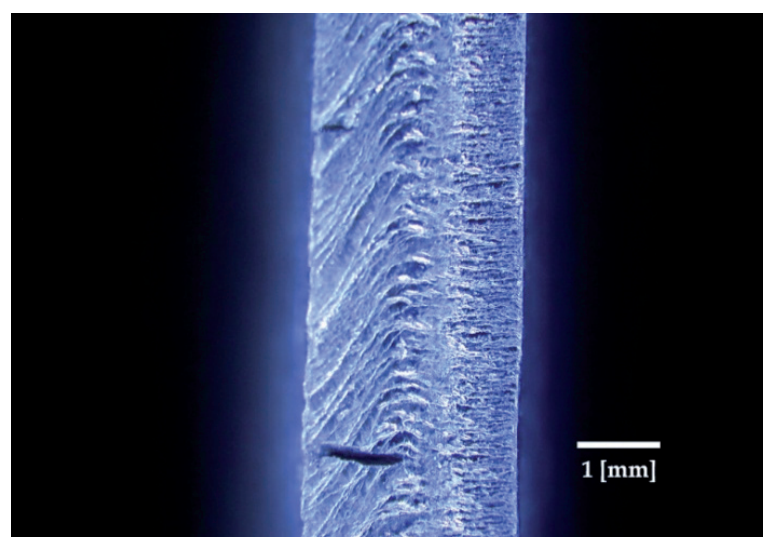

Fig. 13: Micro photography of a $2.5 \mathrm{~mm}$ thick sample with the effect of the over speed processing.

\section{Conclusions}

The best fatigue life probability values were found on the samples processed at a lower cutting speed.

The statistical data has not significantly higher standard deviation, comparing to standard fatigue tests $(s(\log N) \leq 0,15)$.

The cutting speed is correlated with the surface quality. When some pitting flaws or deep micro notches occurred then the scatter of data was higher. Surface flaws have a much more determinant effect on the fatigue life behavior than other factors inherent to the material, as the rolling directions, as both, transversal and longitudinal cold rolled samples, which have similar fatigue resistance values.

The different cutting speeds showed different results after the fatigue test, nevertheless all data of Al 2024 laser machined was within the ASTM specifications for fatigue.

By optimal cutting speed, the $6.0 \mathrm{~mm}$ thick specimens had significant higher fatigue life as results for relative thinner plates between 2.0 to $3.0 \mathrm{~mm}$.

\section{Acknowledgement}

The presented study were supported by the Ministry 
of Education of the Czech Republic by the project MEB 090814.

\section{References}

ASM Aerospace Specification Metals Inc. Database: http://asm.matweb.com/search/SpecificMaterial.asp?bassnum=MA2024T4.

CO2 Laser Processing Technical information, http://www.cz.trumpf. com.

Data Sheets, ASTM STP, No 25, 1985.

ISO 3800:1993, Threaded fasteners -- Axial load fatigue testing -Test methods and evaluation of results.

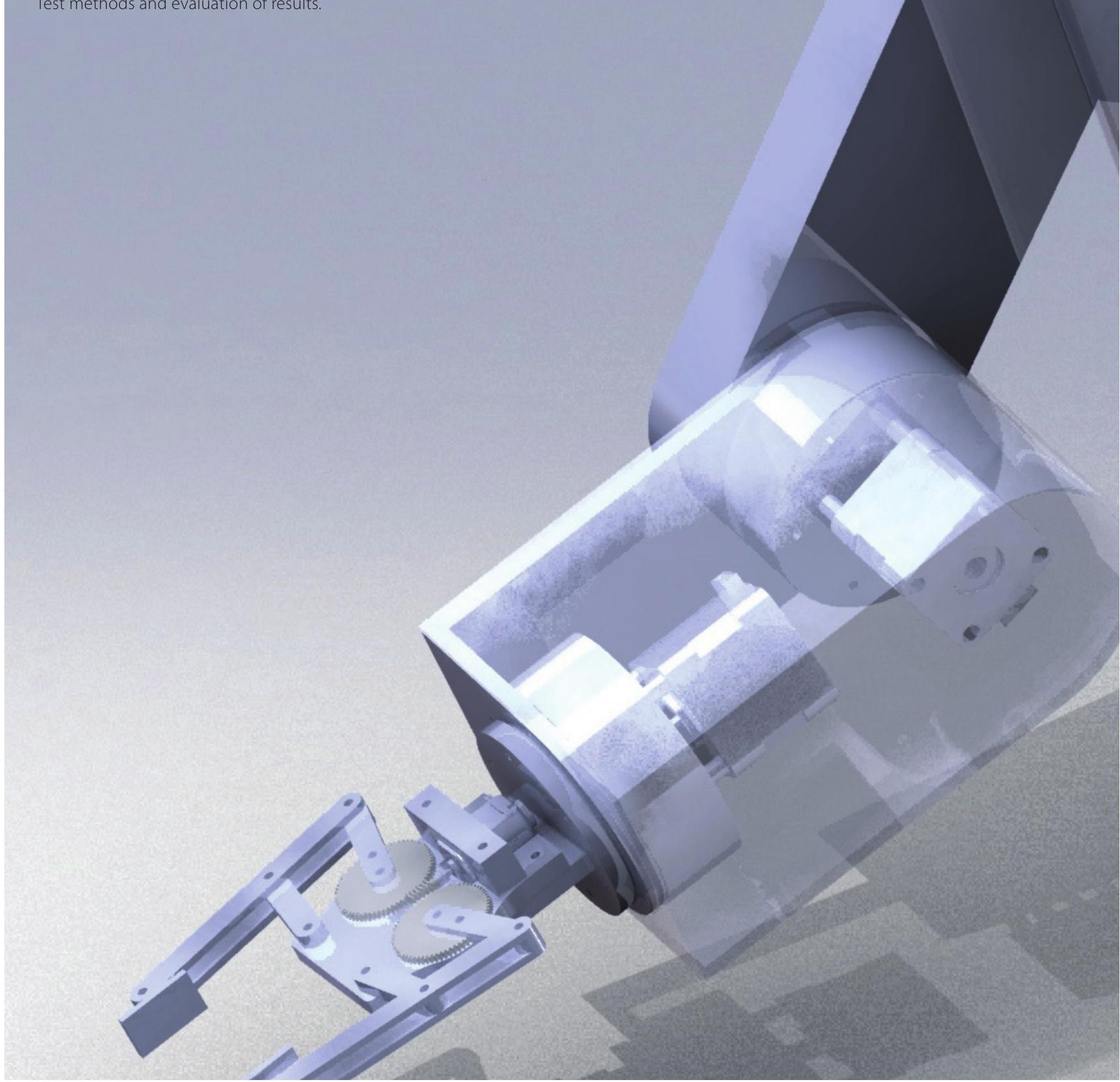

